\title{
A Detected Case of Avian Influenza H9N2 from Influenza-Like Illness Surveillance - Hunan Province, 2020
}

\author{
Siyu Zhang ${ }^{1, \alpha,}$; Chaoyang Huang ${ }^{1,2,2 \times}$; Yingying $\mathrm{Mo}^{3}$; Zhoujian $\mathrm{Wu}^{3}$; Haizhen $\mathrm{Li}^{4}$; \\ Yiwei Huang ${ }^{1,2}$; Fuqiang Liu $^{1, *}$; Lidong Gao ${ }^{1, *}$
}

Jishou Municipal Center for Disease Control and Prevention (Jishou CDC) received a phone call at 7:30 a.m. on April 24, 2020 from Xiangxi Tujia and Miao Autonomous Prefectural CDC (Xiangxi CDC) that avian influenza $\mathrm{A} / \mathrm{H} 9 \mathrm{~N} 2$ virus was detected by quantitative reverse transcription-polymerase chain reaction (RT-PCR) from the throat swab sample of an influenza-like illness in an outpatient in the Xiangxi Traditional Chinese Medicine Hospital.

At 09:00 on April 24, the county and municipal CDCs carried out epidemiological investigations to show that the suspected case was found in a 5-year-old girl. At 03:00 on April 20, the girl developed initial symptoms of fever $\left(37.3^{\circ} \mathrm{C}\right)$, fatigue, abdominal pain, and nausea with vomiting 3 times, and the top body temperature during the course of the disease was $38.6^{\circ} \mathrm{C}$. In the morning of April 20, she was diagnosed with gastrointestinal cold in Xiangxi Traditional Chinese Medicine Hospital, and her throat swab samples were collected by the attending doctor. The fever disappeared by 17:00 the same day. On April 23, Xiangxi CDC carried out a RT-PCR test and the result illustrated that the patient was positive for avian influenza $\mathrm{A} / \mathrm{H} 9 \mathrm{~N} 2$ virus, which verified by retesting on
April 24. The patient was isolated to receive treatment in the hospital since April 25, and was released on April 30 based on the 2 consecutive negative results of RT-PCR test on April 28 and 29 (Figure1).

According to the investigation, the patient had no contact history with similar patients, live poultry, or poultry markets. On April 16, her grandmother bought a live duck from Stall 38 in a market and slaughtered it in Stall 14 before returning home to cook it. The patient's parents and the attending doctor were judged to be close contacts and were placed under medical observation. On April 25, these 3 contacts were sampled and their RT-PCR test results were negative for avian influenza A/H9N2 virus. Up to May 6 , none of them showed symptoms. Throat swab samples were collected from the patient's grandparents and hosts of Stall 38, Stall 14, and adjacent stalls in the market and tested for avian influenza A/H9N2 virus, and all results were negative. About 30 live ducks were sold per day in Stall 38, and these ducks were bought from Huaihua City, Hunan Province. No more H9N2 cases were reported in Xiangxi and Huaihua by the end of May.

To identify the possible source of infection, 172

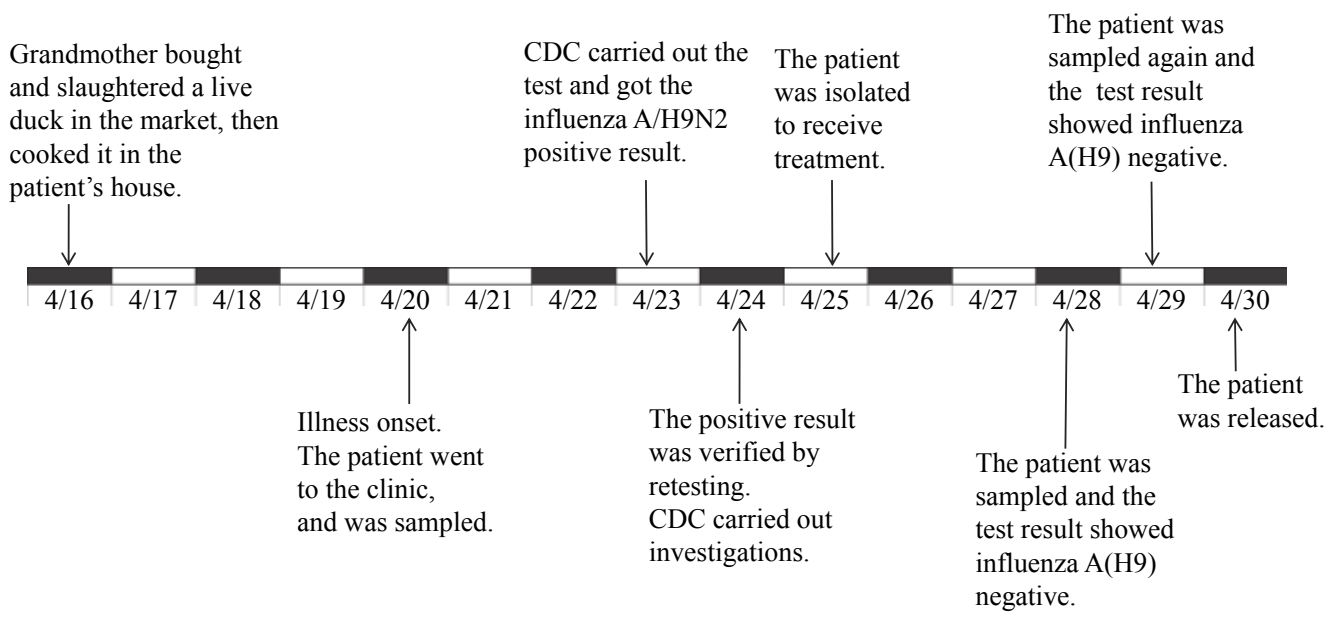

FIGURE 1. Timeline for the epidemiological investigation of the avian influenza A/H9N2 case in Hunan Province, 2020. 
swab samples from live poultry's throat and anus and 26 environmental samples from the market were tested by RT-PCR for subtype H9 influenza virus. A total of 101 live poultry swab samples and 10 environmental samples were influenza A H9 positive, including swabbed samples of cage surface in Stall 38, swabbed samples of chopping boards, and refuse water collected after cleaning poultry in Stall 14. Due to the lack of neuraminidase (NA) test kits, Xiangxi CDC could not identify whether the subtype $\mathrm{H} 9$ positive samples were N2 positive or not.

The full genomes of A/Hunan/11173/2020(H9N2) (HN11173/20) were sequenced. The unrooted phylogenetic tree was generated by the maximum likelihood method using Molecular Evolutionary Genetics Analysis (MEGA, version 7.0.26). The molecular phylogenetic analysis showed that the hemagglutinin (HA) and NA genes of HN11173/20 belonged to the A/Duck/HongKong/Y280/97(H9N2)like lineage of Eurasian branch (Figure 2). The nucleotide sequences homology of 8 segments of HN11173/20 were analyzed with the online Basic Local Alignment Search Tool (BLAST) (Table 1), and matrix protein $(\mathrm{M})$ segment was found to have highly homology with Human H7N9 strain isolated in Changsha (99.51\%); polymerase acidic protein (PA) gene was $98.90 \%$ similar to that of $\mathrm{H} 9 \mathrm{~N} 2$ strain isolated from wildfowl (Accipiter gentilis schvedowi) in Tianjin. HA, NA, nucleoprotein (NP), nonstructural protein (NS), polymerase basic protein 1 (PB1), and polymerase basic protein 2 (PB2) segments were highly homologous with chicken H9N2 strains isolated from Guangdong Province, Shandong Province, and Shanghai Municipality.

The molecular characterization of the HN11173/20 strain were analyzed, whose critical amino acid residues in proteins were the same/similar to those of 3 strains of human $\mathrm{H} 9 \mathrm{~N} 2$ virus previously isolated from Hunan Province (A/Hunan/42088/2017(H9N2), A/Hunan/ $3728 / 2017$ (H9N2), and A/Hunan/43517/2016 (H9N2)), as shown in the Table 2. The cleavage site of HA protein $\mathrm{HA} 1$ and HA2 were SRSSRGL (H3 numbering 334-340), contained the basic amino acid arginine at positions 335 and 338 (R335, R338), which indicated low pathogenicity to poultry. The amino acid of the HA protein of this strain at positions 235-237 (H3 numbered 226-228) was LMG, which susceptibly binded to human-like $\alpha$ 2,6-linked sialic acid receptors and can effectively replicate in mammalian cells (1). NA protein were deleted at the stalk region (positions 63-65), and no oseltamivir associated resistance mutations in amino acid residues were found.

\section{DISCUSSION}

H9N2 influenza virus has low pathogenicity for birds, replicating mainly in the upper respiratory tract and causing mild or no overt signs of illness in specific pathogen-free (SPF) chickens (2). H9N2 infection is usual in live poultry and $\mathrm{H} 9 \mathrm{~N} 2$ contamination is common in poultry-related environment.

The first H9N2 avian influenza case in Hunan Province was found in 2013 (3). As of May 2020, 8 cases have been reported from ILI surveillance, and none had secondary cases. Only two patients had live poultry or live poultry market exposure history. The minimum age was 9 months old, and the maximum age was 15 years old. H9N2 avian influenza virus is most likely to attack minors and cause mild symptoms. Humans, especially exposed populations, are prone to infection.

The surveillance of influenza-like illness in sentinel hospitals played an important role in the discovery of human infected $\mathrm{H} 9 \mathrm{~N} 2$ virus. It is therefore necessary to strengthen the surveillance of sentinel hospitals and live poultry markets to monitor the spread of the influenza A viruses.

After analyzing the biomolecular characteristics of HN11173/20 strain, we observed the virulence of this isolate was relatively weak, and it had the characteristics of low-pathogenic avian influenza (LPAI) virus. The genome sequence analysis showed that the cleavage site of its HA was SRSSR $\downarrow$ GL, without multiple continuous basic amino acids insertion, which also conformed to the characteristics of the LPAI virus. HN11173/20 strain contained the amino acid leucine at position 226 in its HA receptorbinding sites, which had the characteristics to susceptibly bind to receptors of human cells and transmit directly from birds to humans causing mild flu-like symptoms. Online BLAST alignment showed that the $\mathrm{M}$ fragment of this strain had the most homology with H7N9 strain isolated from Changsha in 2017, suggesting that the reassortment of H9N2 and $\mathrm{H} 7 \mathrm{~N} 9$ could produce $\mathrm{H} 7 / \mathrm{H} 9$ subtype avian influenza virus strains which could infect humans directly.

The prevalence of avian influenza viruses among 

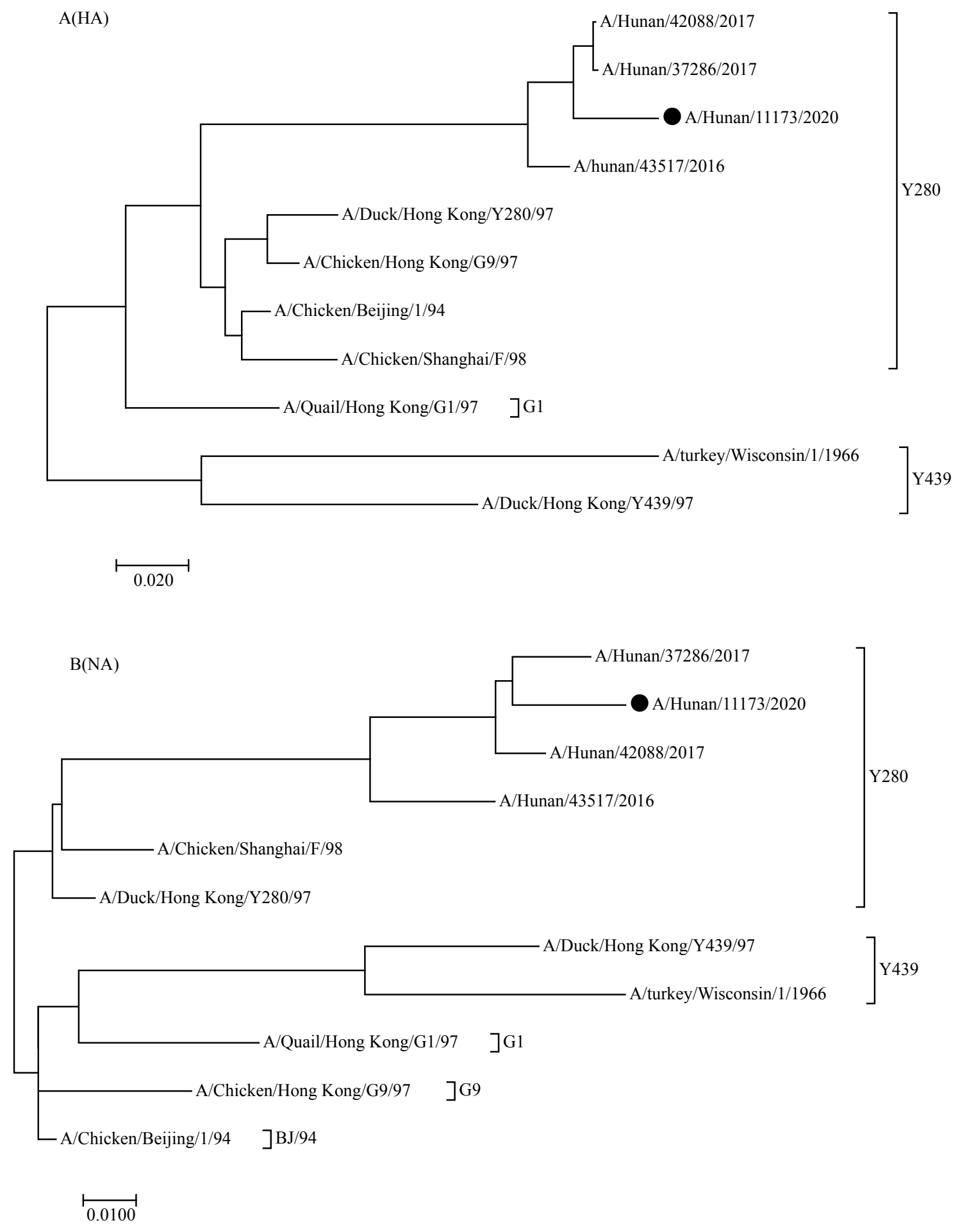

FIGURE 2. Phylogenetic trees of hemagglutinin (HA) and neuraminidase (NA) gene segments of $H N 11173 / 20$ isolated from Hunan Province. (A) HA gene segments; (B) NA gene segments.

poultry and the emerging of sporadic human infected case pose a continuous threat to public health. This work reported a new avian influenza virus H9N2 infected case in Hunan Province. The phylogenetic features and the potential pathogenesis-related amino acid sites were analyzed. This work provided a pivotal reference for the understanding of the human infection of avian influenza viruses and points out the importance of the surveillance of the virus based on a One-Health strategy.

Acknowledgments: We thank the colleagues from the Chinese National Influenza Center, who isolated 
TABLE 1. Homology of 8 segments of HN11173/20 analyzed with the online Basic Local Alignment Search Tool.

\begin{tabular}{|c|c|c|c|c|c|c|}
\hline Segment & $\begin{array}{l}\text { Sequence length } \\
\text { (bp) }\end{array}$ & $\begin{array}{l}\text { The highest nucleotide identity of } \\
\text { H9N2 virus in GenBank }\end{array}$ & $\begin{array}{l}\text { Accession } \\
\text { ID }\end{array}$ & Collection date & $\begin{array}{l}\text { Collecting } \\
\text { location }\end{array}$ & Identities \\
\hline $\mathrm{HA}$ & 1,742 & A/chicken/China/C7/2018(H9N2) & MN384772 & Jul-2018 & Guangdong & $98.50 \%$ \\
\hline NA & 1,442 & A/chicken/China/63/2019(H9N2) & MN263217 & 05-Jun-2019 & Shanghai & $98.54 \%$ \\
\hline NP & 1,565 & A/chicken/China/1103/2019(H9N2) & MN918142 & 20-Nov-2019 & Shanghai & $98.27 \%$ \\
\hline NS & 890 & A/chicken/Shandong/3424/2016(H9N2) & MH667576 & 02-Jun-2016 & Shandong & $98.76 \%$ \\
\hline MP & 1,027 & A/Hunan Changsha/26/2017(H7N9) & MF370250 & 04-Feb-2017 & Hunan & $99.51 \%$ \\
\hline PA & 2,175 & $\begin{array}{l}\text { A/Accipiter gentilis schvedowi/ } \\
\text { Tianjin/22/2017(H9N2) }\end{array}$ & MH114054 & 15-Jun-2017 & Tianjin & $98.90 \%$ \\
\hline PB1 & 2,341 & A/chicken/China/C7/2018(H9N2) & MN384772 & Jul-2018 & Guangdong & $98.76 \%$ \\
\hline PB2 & 2,339 & A/chicken/Shandong/3424/2016(H9N2) & MH667576 & 02-Jun-2016 & Shandong & $97.52 \%$ \\
\hline
\end{tabular}

Abreviation: $\mathrm{HA}=$ hemagglutinin; $\mathrm{NA}=$ neuraminidase; $\mathrm{NP}=$ nucleoprotein; NS=nonstructural protein; $\mathrm{MP}=\mathrm{matrix}$ protein; $\mathrm{PA}=$ polymerase acidic protein; $\mathrm{PB}=$ polymerase basic protein.

*The date of this BLAST search was Jun 12, 2020.

TABLE 2. The molecular characteristics of the H9N2 influenza viruses isolated from Hunan Province.

\begin{tabular}{|c|c|c|c|c|c|c|c|c|c|c|c|c|c|c|c|}
\hline \multirow{3}{*}{ Virus } & \multicolumn{7}{|c|}{ HA (H3 Numbering) } & \multirow{3}{*}{$\begin{array}{c}\text { NA } \\
\text { deletion } \\
63-65\end{array}$} & \multirow{3}{*}{\begin{tabular}{r|} 
M1 \\
15
\end{tabular}} & \multirow{3}{*}{$\begin{array}{l}\text { M2 } \\
28\end{array}$} & \multirow{2}{*}{\multicolumn{2}{|c|}{ NS1 PB1 }} & \multirow{2}{*}{\multicolumn{3}{|c|}{ PA }} \\
\hline & \multicolumn{6}{|c|}{ Receptor binding site } & \multirow{2}{*}{$\begin{array}{c}\begin{array}{c}\text { Cleavage } \\
\text { site }\end{array} \\
334-340\end{array}$} & & & & & & & & \\
\hline & 158 & 183 & 190 & 226 & 227 & 228 & & & & & 92 & 227 & 13 & 356 & 409 \\
\hline A/Hunan/11173/2020(H9N2) & $\mathrm{N}$ & $\mathrm{N}$ & $\mathrm{T}$ & $\mathrm{L}$ & $M$ & G & SRSSRGL & Yes & 1 & $\mathrm{~V}$ & $\mathrm{D}$ & $\mathrm{K}$ & $P$ & $\mathrm{R}$ & $\mathrm{N}$ \\
\hline $\begin{array}{l}\text { The other three H9N2 viruses previously } \\
\text { isolated from Hunan Province }\end{array}$ & $\mathrm{N}$ & $\mathrm{N}$ & $\mathrm{T}$ & L & M & G & SRSSRGL & Yes & 1 & $\mathrm{~V} / \mathrm{I}$ & $\mathrm{D}$ & $\mathrm{K}$ & $P$ & $\mathrm{R}$ & $\mathrm{N}$ \\
\hline
\end{tabular}

Abreviation: N=Aspara; T=Threonine; L=Leucine; M=Methionine; G=Glycine; S=Serine; R=Arginine; I=Isoleucine; V=Valine; $D=A s p a r t i c$ acid; $\mathrm{K}=$ Lysine; $\mathrm{P}=$ Proline.

and sequenced the full genome of $\mathrm{A} / \mathrm{Hunan} / 11173 /$ 2020(H9N2).

doi: $10.46234 / \mathrm{ccdcw} 2020.170$

\# Corresponding authors: Fuqiang Liu, 88037558@qq.com; Lidong Gao, gldlj@hotmail.com.

\footnotetext{
Hunan Provincial Center for Disease Control and Prevention, Changsha, Hunan, China; ${ }^{2}$ Hunan Provincial Key Laboratory of Microbial Molecular Biology, Changsha, Hunan, China; ${ }^{3}$ Xiangxi Tujia and Miao Autonomous Prefectural Center for Disease Control and Prenvention, Jishou, Hunan, China; ${ }^{4}$ Jishou Municipal Center for Disease Control and Prevention, Jishou, Hunan, China.

\& Joint first authors.

Submitted: June 20, 2020; Accepted: July 14, 2020
}

\section{REFERENCES}

1. Wan HQ, Perez DR. Amino acid 226 in the hemagglutinin of H9N2 influenza viruses determines cell tropism and replication in human airway epithelial cells. J Virol 2007;81(10):5181 - 91. http://dx.doi.org/ 10.1128/JVI.02827-06.

2. Guo YJ, Krauss S, Senne DA, Mo IP, Lo KS, Xiong XP, et al. Characterization of the pathogenicity of members of the newly established H9N2 influenza virus lineages in Asia. Virology 2000; 267(2):279 - 88. http://dx.doi.org/10.1006/viro.1999.0115.

3. Zhang SY, Huang YW, Hu SX, Zhang HJ, Sun QL, Deng ZH, et al. Epidemiologic characteristics of human avian influenza in Hunan Province from 2005 to 2017. Chin J Dis Control Prev 2018;22(10): 1037 - 40. http://dx.doi.org/10.16462/j.cnki.zhjbkz.2018.10.014. (In Chinese). 